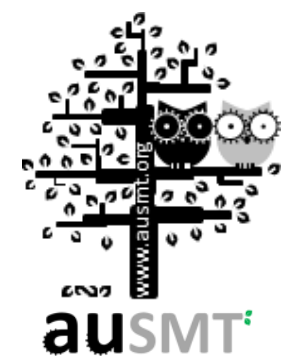

\title{
Using Red-Emitting Ca2Si5N8Eu2+ Phosphor for Improving Color Uniformity and Color Quality Scalle of the in-cup Packaging MCW-LEDs
}

\section{Tran Thanh Nam1, Tran Hoang Quang Minh',*, and Le Anh Vu' ${ }^{1}$}

${ }^{1}$ Optoelectronics Research Group, Faculty of Electrical and Electronics Engineering, Ton Duc Thang University, Ho Chi Minh City, Vietnam (Received 1 April 2017; Accepted 25 May 2017; Published online 1 March 2018)

*Corresponding author: tranhoangquangminh@tdt.edu.vn

DOI: 10.5875 /ausmt.v8i1.1389

\begin{abstract}
Light emitting diodes (LEDs) are commonly used in general lighting applications due to their rapidly improving efficiency, long-life, compact, low power consumption, and high climate resistance. In this paper, the Red-Emitting $\mathrm{Ca}_{2} \mathrm{Si}_{5} \mathrm{~N}_{8} \mathrm{Eu}^{2+}$ Phosphor was investigated like an innovative approach for improvement on the color uniformity and Color Quality Scale (CQS) of $8500 \mathrm{~K}, 7700 \mathrm{~K}, 7000 \mathrm{~K}$ multi-chip white LED lamps (MCW-LEDs) with the in-cup packaging. The influence of the Red-Emitting $\mathrm{Ca}_{2} \mathrm{Si}_{5} \mathrm{~N}_{8} \mathrm{Eu}^{2+}$ Phosphor concentration on the color uniformity and the CQS is simulated, analyzed, and investigated with using Mat lab and LightTools software. The research results indicated that the color uniformity and the CQS were crucially affected by the Red-Emitting $\mathrm{Ca}_{2} \mathrm{Si}_{5} \mathrm{~N}_{8} \mathrm{Eu}^{2+}$ Phosphor concentration. This paper provided an important recommendation for selecting and developing the phosphor materials for the in-cup packaging MCW-LEDs manufacturing in the near future.
\end{abstract}

Keywords: the Red-Emitting $\mathrm{Ca}_{2} \mathrm{Si}_{5} \mathrm{~N}_{8} \mathrm{Eu}^{2+}$ Phosphor; MCW-LEDs; Color Quality Scale; the color uniformity

\section{Introduction}

In the last four decades, light emitting diodes (LEDs) with compound semiconductor technology had a rapid development trend. Since the first red LEDs that was invented by Holonyak and Bevacqua in 1962 [1], considerable efforts have been put into the study to obtain brighter LEDs. In the decades that followed, LEDs were used extensively in digital displays and signaling applications. However, only around 1995 high brightness and blue LEDs were developed, which made it possible to use LEDs for general lighting. Nowadays, MCW-LEDs have attracted considerable attention from both manufacturers and consumers of the general lightings due to its excellent properties for lighting, including high brightness, low power consumption, long lifetime, fast response as well as climate impact resistance [2]. LEDs have a narrow emission spectrum. In a popular way, there are three different approaches which can be used for generating white light based on LEDs. In the first method, the white light is getting by mixing reds, greens, and blues, i.e. red- green-blue (RGB) LEDs. By using an ultraviolet (UV) LED to stimulate RGB phosphors in the second method, and by using a blue-emitting diode that excites a yellow-emitting phosphor embedded in the epoxy dome; the combination of blue and yellow light makes a white-emitting LED) in the third one. The last method is widely used in general lighting applications for white LEDs because of its simple procedure [3-7].

Nowadays, the improvement of optical properties of WLEDs is main direction research in optoelectronics. Up to date, many studies focused on improving optical properties of WLEDs by controlling geometry, thickness, concentration, and packaging of the yellow-emitting phosphor in the phosphor compounding of WLEDs [8-14]. In another way, some researchers just concentrated on adding $\mathrm{SiO}_{2}$ to control and enhance the optical properties of WLEDs $[15,16]$. Previous studies showed that in this way the optical properties of WLEDs could be improved significantly. These studies just focused on single chip WLEDs with the low correlated temperature or only control one of three optical properties of WLEDs (the color correlated temperature deviation (D-CCT), CRI and 
luminous efficacy). However, far too little attention has been paid to improving the optical properties of the high color temperature MCW-LEDs yet. This research could fully fill the remaining gap.

In this paper, the red-emitting $\mathrm{Ca}_{2} \mathrm{Si}_{5} \mathrm{~N}_{8} \mathrm{Eu}^{2+}$ phosphor $[17,18]$ is proposed to improve the optical properties (color quality scale (CQS) and color uniformity) of the in-cup package multichip WLEDs (MCW-LEDs) with the high CCT at $7000 \mathrm{~K}, 7700 \mathrm{~K}$, and $8500 \mathrm{~K}$. The research results, using the simulation package Light Tool and Mat lab software based on the Lambert-Beer law and Mie theory, demonstrated that concentration of the redemitting $\mathrm{Ca}_{2} \mathrm{Si}_{5} \mathrm{~N}_{8} \mathrm{Eu}^{2+}$ phosphor had significant impact on the color uniformity (D-CCT) and CQS of the in-cup packaging MCW-LEDs. The overall structure of the study takes the form of three segments. In the first segment, the MCW-LEDs physical model having average CCT of $8500 \mathrm{~K}$, $7700 \mathrm{~K}$, and $7000 \mathrm{~K}$ is conducted by the commercial Light Tools software. Secondly, by varying concentration of the red-emitting $\mathrm{Ca}_{2} \mathrm{Si}_{5} \mathrm{~N}_{8} \mathrm{Eu}^{2+}$ phosphor particles, the D-CCT and CQS calculated, analyzed and investigated. Finally, according to the results, the Beer-Lambert law, and Mie theory, the effect of the red-emitting $\mathrm{Ca}_{2} \mathrm{Si}_{5} \mathrm{~N}_{8} \mathrm{Eu}^{2+}$ phosphor particles on CQS and D-CCT of MCW-LEDs is discussed and demonstrated totally. From the results, the red-emitting $\mathrm{Ca}_{2} \mathrm{Si}_{5} \mathrm{~N}_{8} \mathrm{Eu}^{2+}$ phosphor could be a prospective approach for MCW-LEDs improvement in the near future.

\section{Optical and Simulation Model MCW-LEDs}

The simulations were carried out using the commercial software package LightTools. The simulation comprise the setup of the conformal phosphor package with average CCT of $8500 \mathrm{~K}, 7000 \mathrm{~K}$, and $5600 \mathrm{~K}$. We use the real-world model of MCW-LEDs (Fig. 1(a)) to guarantee that our simulation results reflect precisely the impact of our considered parameter (concentration of the

LE Anh Vu is member of Optoelectronics Research Group Faculty of Electricals and Electronic Engineering, Ton Duc Thang University-Ho Chi Minh City, Viet Nam. He received his BS in Electronics and Telecommunications from $\mathrm{Ha}$ No University of Technology, Mater and PHD degrees in Electronics and Electric from the Dongguk University in 2007 and 2012, respectively. From 2015 to 2017 he was a postdoc fellow at Korea Institute of Science and Technology. His current research interests include Robotics vision, human detection feature matching, 3D video and optoelectronics.

TRAN Hoang Quang Minh (corresponding author) defended his Ph.D. thesis at Tomsk Polytechnic University, Tomsk, Russian Federation. The author's major fields of study are High-voltage Power System, Relay Protections and Optoelectronics. He is working as Lecturer in Faculty of Electrical and Electronics Engineering, Ton Duc Thang University, Ho Chi Minh City, Vietnam.

TRAN Thanh Nam is at Optoelectronics Research Group - Faculty of Electricals and Electronic Engineering, Ton Duc Thang University-Ho Chi Minh City, Viet Nam. He received his BS in Advanced Materials from Ho Chi Minh City University of Technology (Vietnam) in 2008, and M.Sc degree in Microsystem from Vestfold University College (Norway) in 2011. He is now pursuing his PhD degree at Norwegian University of Science and Technology (Norway) focusing on semiconductor laser diodes, especially GaSb-based type I laser diode fo gas sensing applications. red-emitting $\mathrm{Ca}_{2} \mathrm{Si}_{5} \mathrm{~N}_{8} \mathrm{Eu}^{2+}$ phosphor) and are not biased by other factors such as LED's wavelength, waveform, light intensity, and operating temperature. This model possesses the best optical-thermal stability, hence, can minimize the variations caused by uninterested parameters. Secondly, we set the depth, the inner and outer radius of the reflector to $2.07 \mathrm{~mm}, 8 \mathrm{~mm}$ and 9.85 $\mathrm{mm}$, respectively. Nine LED chips are covered with fixed thickness of $0.08 \mathrm{~mm}$ and $2.07 \mathrm{~mm}$. Each blue chip has a dimension of $1.14 \mathrm{~mm}$ by $0.15 \mathrm{~mm}$, the radiant flux of 1.16 $\mathrm{W}$, and the peak wavelength of $453 \mathrm{~nm}$. Fig. 1(b) shows that the phosphor layer of the in-cup phosphor packaging is coated conformally on 9 LEDs. These phosphor layers consist of the yellow-emitting YAG:Ce and the redemitting $\mathrm{Ca}_{2} \mathrm{Si}_{5} \mathrm{~N}_{8} \mathrm{Eu}^{2+}$ phosphors particles and the silicone glue, which respectively have the refractive indices of 1.83 , 1.93 and 1.50. Also, the average radius of YAG:Ce phosphor particles are set to $7.25 \mu \mathrm{m}$ for all packages, a value of real particle size. On purpose to make the color of MCW-LEDs produce the same when the concentration of the red-emitting $\mathrm{Ca}_{2} \mathrm{Si}_{5} \mathrm{~N}_{8} \mathrm{Eu}^{2+}$ phosphor varies, the yellowemitting YAG:Ce phosphor concentration should be inversely changed to provide same CCT value. The weight percentage of the MCW-LEDs phosphor layers can be expressed as the below equation:

$$
\sum \mathrm{W}_{\mathrm{pl}}=\mathrm{W}_{\text {Yellow phosphor }}+\mathrm{W}_{\text {silicone }}+\mathrm{W}_{\text {Red phosphor }}=100 \%
$$

Here the $W_{\text {silicone }}, W_{\text {Yellow phosphor }}$ and $W_{\text {Red phosphor }}$ are in turn the weight percentage of the silicone glue, the yellow-emitting YAG: Ce phosphor and the red-emitting CeTb phosphors [22].

\section{Results and Discussion}

In this segment, the color correlated temperature deviation (D-CCT) and the CQS of the in-cup packaging $8500 \mathrm{~K}, 7700 \mathrm{~K}$, and $7000 \mathrm{~K} \mathrm{MCW}$-LEDs are calculated and obtained by using the commercial software Light Tools. In this simulation, the concentration of the red-emitting $\mathrm{Ca}_{2} \mathrm{Si}_{5} \mathrm{~N}_{8} \mathrm{Eu}^{2+}$ phosphor varied continuously from $0 \%$ to $0.32 \%$. From the simulation results, the corresponding values of D-CCT are calculated and indicated in Fig. 2. As shown in Fig, 2, the D-CCT had a slight decrease when the concentration of the red-emitting $\mathrm{Ca}_{2} \mathrm{Si}_{5} \mathrm{~N}_{8} \mathrm{Eu}^{2+}$ phosphor varied from $0 \%$ to $0.24 \%$. After that, the D-CCT of the MCW-LEDs increased while the red-emitting $\mathrm{Ca}_{2} \mathrm{Si}_{5} \mathrm{~N}_{8} \mathrm{Eu}^{2+}$ phosphor changed to $0.32 \%$. The most interesting aspect of this graph is the lowest value of the D-CCT is obtained at $0.16-0.24 \%$ concentration of the red-emitting $\mathrm{Ca}_{2} \mathrm{Si}_{5} \mathrm{~N}_{8} \mathrm{Eu}^{2+}$ phosphor. On the other way, Fig. 3 shows the CQS of the conformal packaging MCW-LEDs while the concentration of the red phosphor changed from $0 \%$ to 
$0.32 \%$. It can be indicated that the CQS grows with the concentration red phosphor in the range from $0 \%$ to nearly $0.32 \%$. The highest CQS is obtained with the red weight vary from $0.16 \%$ to $0.24 \%$. In Fig. 3 , the CQS could be obtained highest value near 72 . From the results, the CQS of high CCT MCW-LEDs can be controlled by adding red-emitting phosphor in phosphor compound and varying its concentration. The best value of the D-CCT and CQS can be obtained when the concentration of the redemitting $\mathrm{Ca}_{2} \mathrm{Si}_{5} \mathrm{~N}_{8} \mathrm{Eu}^{2+}$ phosphor is nearly $0.24 \%$.

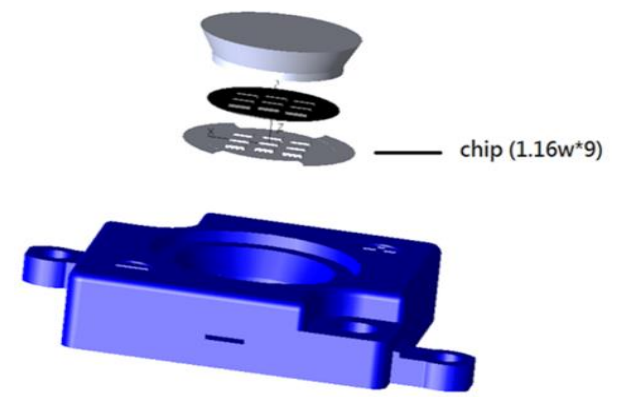

(a)

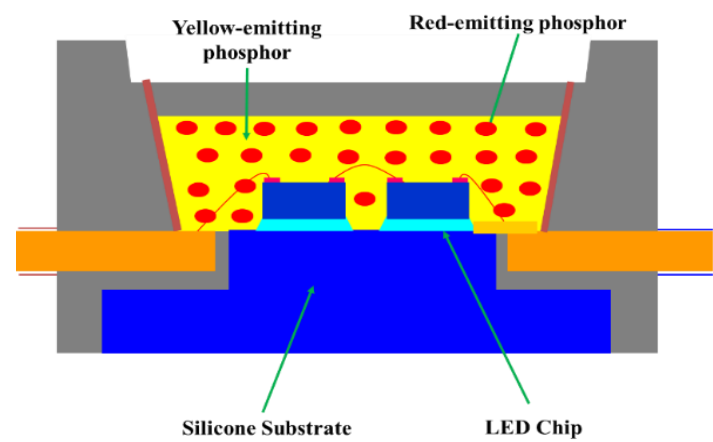

(b)

Figure 1. (a) The MCW-LED product of the Siliconware Precision Industries Co., Ltd., Taiwan, (b), Illustration of MCW-LEDs with the conformal phosphor package.

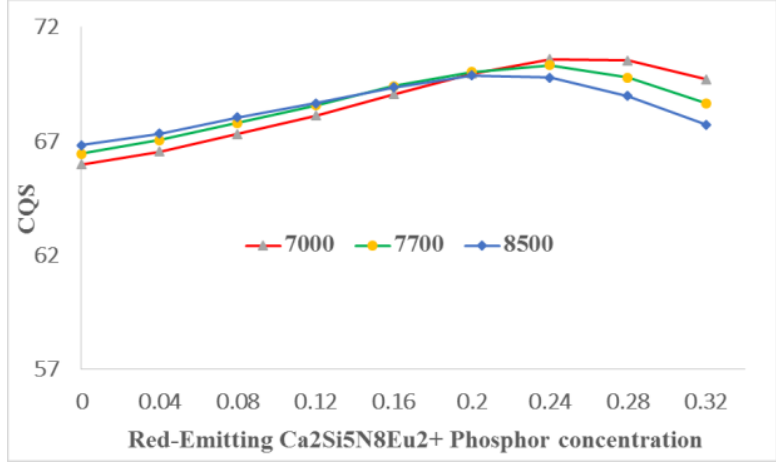

Figure 2. The color quality scale (CQS) at average CCTs of $8500 \mathrm{~K}, 7700$ $\mathrm{K}$, and $7000 \mathrm{~K}$.

The influence of the concentration of the redemitting $\mathrm{Ca}_{2} \mathrm{Si}_{5} \mathrm{~N}_{8} \mathrm{Eu}^{2+}$ phosphor on the color uniformity (D-CCT) and the CQS can be demonstrated by using Mie theory [19]. Here, we can apply Mie-scattering theory [20, $21]$, the scattering coefficient $\mu_{s c a}(\lambda)$, anisotropy factor $g(\lambda)$, and reduced scattering coefficient $\delta_{\text {sca }}(\lambda)$ can be computed by the below expressions (1), (2), and (3) [22]:

$$
\begin{gathered}
\mu_{s c a}(\lambda)=\int N(r) C_{s c a}(\lambda, r) d r \\
g(\lambda)=2 \pi \iint_{-1}^{1} p(\theta, \lambda, r) f(r) \cos \theta d \cos \theta d r \\
\delta_{s c a}=\mu_{s c a}(1-g)
\end{gathered}
$$

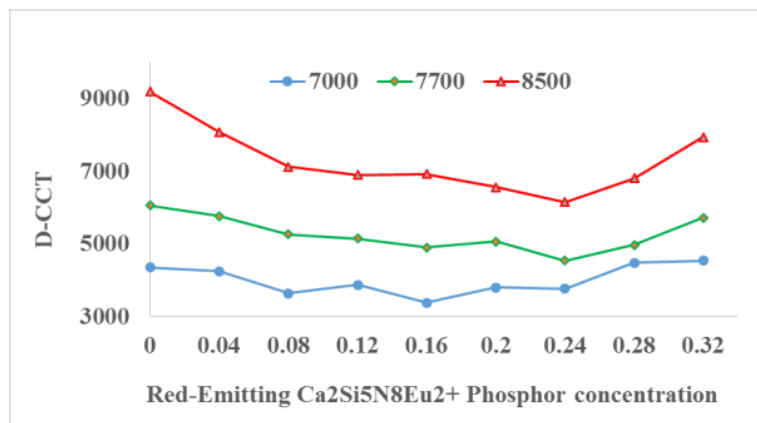

Figure 3. The D-CCT at average CCTs of $8500 \mathrm{~K}, 7700 \mathrm{~K}$, and 7000K.

Where $N(r)$ is the density distribution of diffusional particles (per cubic millimeter), millimeter)

$C_{s c a}$ is the scattering cross sections (per square

$p(\vartheta, \lambda, r)$ is the phase function,

$\lambda$ is the wavelength of the incident light (nanometers),

$r$ is the radius of particles (micrometers),

$\vartheta$ is the scattering angle (degree),

$f(r)$ is the size distribution function of the diffusers in the phosphor layer.

$$
\begin{aligned}
& f(r)=f_{\text {dif }}(r)+f_{\text {phos }}(r) \\
N(r)= & N_{\text {dif }}(r)+N_{\text {phos }}(r) \\
= & K_{N} \cdot\left[f_{\text {dif }}(r)+f_{\text {phos }}(r)\right]
\end{aligned}
$$

$N_{\text {dif }}(r)$ is the diffusive particle number density,

$N_{\text {phos }}(r)$. Is the phosphor particle number density,

$f_{\text {dif }}(r)$ and $f_{\text {phos }}(r)$ are the size distribution function data of the diffusor and phosphor particle,

$K_{N}$ is the number of the unit diffusor for one diffuser concentration and $K_{N}$ can be obtained by:

$$
c=K_{N} \int M(r) d r
$$

Where $M(r)$ is the mass distribution (milligrams) of the unit diffusor. Below equation can calculate $M(r)$ :

$$
M(r)=\frac{4}{3} \pi r^{3}\left[\rho_{\text {dif }} f_{\text {dif }}(r)+\rho_{\text {phos }} f_{\text {phos }}(r)\right]
$$



LEDS

$\rho_{\text {diff }}(r)$ and $\rho_{\text {phos }}(r)$ are the density of diffuser and phosphor crystal.

In Mie theory, $C_{\text {sca }}$ is calculated by:

$$
C_{s c a}=\frac{2 \pi}{k^{2}} \sum_{0}^{\infty}(2 n-1)\left(\left|a_{n}\right|^{2}+\left|b_{n}\right|^{2}\right)
$$

Where $k$ is the wavenumber $(2 \pi / \lambda)$,

$a_{n}$ and $b_{n}$ are the expansion coefficients with even symmetry and odd symmetry, respectively. These coefficients can be calculated by below equations:

$$
\begin{gathered}
a_{n}(x, m)=\frac{\psi_{n}^{\prime}(m x) \psi_{n}(x)-\mathrm{m} \psi_{n}(m x) \psi_{n}^{\prime}(x)}{\psi_{n}^{\prime}(m x) \xi_{n}(x)-\mathrm{m} \psi_{n}(m x) \xi_{n}^{\prime}(x)} \\
a_{n}(x, m)=\frac{m \psi_{n}^{\prime}(m x) \psi_{n}(x)-\psi_{n}(m x) \psi_{n}^{\prime}(x)}{m \psi_{n}^{\prime}(m x) \xi_{n}(x)-\psi_{n}(m x) \xi_{n}^{\prime}(x)}
\end{gathered}
$$

Where $x$ is the size parameter (=k.r),

$m$ is the refractive index of the scattering diffusive particles,

$\psi_{n}(x)$ and $\xi_{n}(x)$ are the Riccati-Bessel function.

The extinction coefficient values of the red phosphor are verified for two distinct wavelengths, 555 $\mathrm{nm}$ and $453 \mathrm{~nm}$, which are the emission peaks of the YAG:Ce phosphor and the LED chips, respectively. The variation of the mentioned parameters on the red phosphor concentration according to the above equations are displayed in Fig.4 (a)-(c) for $8500 \mathrm{~K}, 7000 \mathrm{~K}$ and $5600 \mathrm{~K}$ respectively.

As displayed in Fig. 5, the scattering coefficients grew with increasing red phosphor concentration. It means that the white-light quality can be enhanced by controlling red phosphor concentration. The reduced scattering coefficient of red phosphor with wavelengths $453 \mathrm{~nm}, 555 \mathrm{~nm}$ and $680 \mathrm{~nm}$ are approximate with each other (Fig 6). It indicated that the scattering stability of red phosphor is useful for controlling the color quality of MCW-LEDs. The results indicated that red phosphor particles had a significant advantage in producing blue light. The more the blue light emitted, the more the yellow ring phenomenon reduced. These calculations and analysis can clearly use to proving the results in Fig. 2 and Fig. 3 [22].

\section{Conclusion}

The purpose of the current study was to determine the influence of the red-emitting $\mathrm{Ca}_{2} \mathrm{Si}_{5} \mathrm{~N}_{8} \mathrm{Eu}^{2+}$ phosphor concentration on the color uniformity and the CQS of the in-cup packaging MCW-LEDs. The results of this investigation show that the concentration of the red phosphor particles crucial influenced on the optical properties of the in-cup packaging MCW-LEDs. The both $\mathrm{D}-\mathrm{CCT}$ and CQS can obtain the best value at the 0.24$0.28 \%$ concentration of the red-emitting $\mathrm{Ca}_{2} \mathrm{Si}_{5} \mathrm{~N}_{8} \mathrm{Eu}^{2+}$ phosphor. Further studies need to be carried out to validate the influence of the red-emitting $\mathrm{Ca}_{2} \mathrm{Si}_{5} \mathrm{~N}_{8} \mathrm{Eu}^{2+}$ phosphor size on the optical properties of the conformal packaging MCW-LEDs.

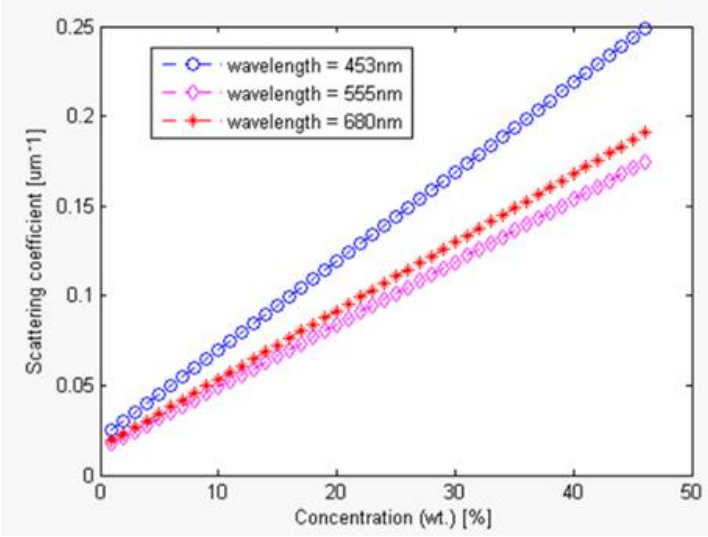

Figure 4. Scattering coefficients of $\mathrm{Ca}_{2} \mathrm{Si}_{5} \mathrm{~N}_{8} \mathrm{Eu}^{2+}$ in proportion to wavelengths of $453 \mathrm{~nm}, 555 \mathrm{~nm}$, and $680 \mathrm{~nm}$.

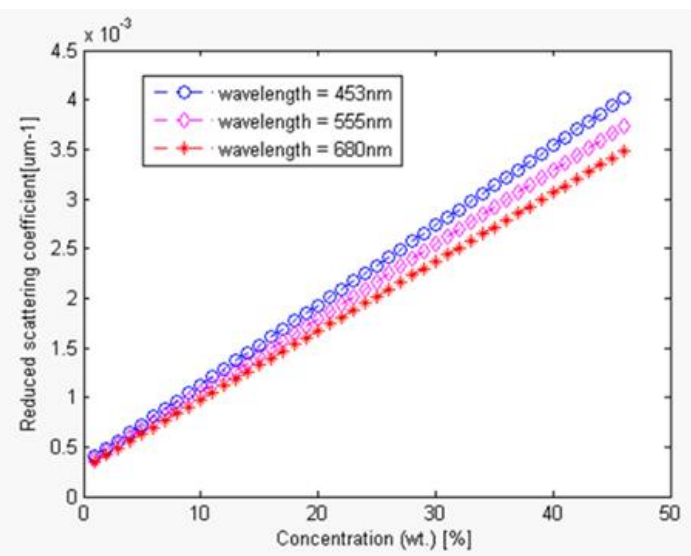

Figure 5. Reduced scattering coefficient of $\mathrm{Ca}_{2} \mathrm{Si}_{5} \mathrm{~N}_{8} \mathrm{Eu}^{2+}$ with wavelengths of $453 \mathrm{~nm}, 555 \mathrm{~nm}$, and $680 \mathrm{~nm}$.

\section{Acknowledgements}

The authors appreciate the support of Professor Hsiao-Yi Lee, Department of Electrical Engineering, National Kaohsiung University of Applied Sciences, Kaohsiung, Taiwan.

\section{References}

[1] J. N. Holonyak and S. F. Bevacqua, "Coherent (Visible) Light Emission from Ga (As1 - xPx) Junctions," Applied Physics Letters, vol. 1, no. 4 , pp. 82-83, 1962. doi: $10.1063 / 1.1753706$

[2] Schubert, E. Fred. "History of Light-Emitting Diodes," 
Light-Emitting Diodes, Cambridge University Press, 2006, pp. 1-26.

doi: $10.1017 /$ cbo9780511790546.002

[3] Schubert, "White Inorganic LEDs," Fundamentals of Solid-State Lighting, 2014, pp. 261-276.

[4] S. Liu and X.-B. Luo, LED Packaging for Lighting Applications: Design, Manufacturing and Testing, Chemical Industry Press and John Wiley \& Sons, Beijing, 2011. doi: $\underline{10.1002 / 9780470827857}$

[5] Nadarajah Narendran, Lei Deng, Richard M. Pysar, Yimin Gu, and Hua Yu, "Performance Characteristics of High-Power Light-Emitting Diodes," in proceeding of the Third International Conference on Solid State Lighting, San Diego, California, January 26, 2004. doi: $10.1117 / 12.515647$

[6] H. Yamamoto and T. Yamamoto, "Phosphors for White LEDs," Nitride Semiconductor Light-Emitting Diodes (LEDs), 2014, pp. 144-180. doi: $10.1533 / 9780857099303.1 .144$

[7] S. Ye, F. Xiao, Y. Pan, Y. Ma, And Q. Zhang, "Phosphors in Phosphor-Converted White Light-Emitting Diodes: Recent Advances in Materials, Techniques, and Properties," Materials Science and Engineering: R: Reports, vol. 71, no. 1, pp. 1-34, 2010. doi: 10.1016/j.mser.2010.07.001

[8] C.-C. Sun, Y.-Y. Chang, and G. H. Yang, "Exploring Packaging Efficiency in Phosphor-Converted White LEDs," SPIE Newsroom, April, 2015. doi: $10.1117 / 2.1201502 .005739$

[9] Z.-Y. Liu, S. Liu, K. Wang, and X.-B. Luo, "Studies on Optical Consistency of White LEDs Affected by Phosphor Thickness and Concentration Using Optical Simulation," IEEE Transactions on Components and Packaging Technologies, vol. 33, no.4, pp. 680-687, 2010. doi: $10.1109 /$ tcapt.2010.2044576

[10] R.-Y. Yu, S.-Z. Jin, S.-Y. Cen, and P. Liang, "Effect of the Phosphor Geometry on the Luminous Flux of Phosphor-Converted Light-Emitting Diodes," IEEE Photonics Technology Letters, vol. 22, no. 23, pp. 1765-1767, 2010. doi: 10.1109/LPT.2010.2085080

[11] J.-P. You, Y.-H. Lin, Nguyen T. Tran, and Frank G. Shi, "Phosphor Concentration Effects on Opto-thermal Characteristics of Phosphor-Converted White LightEmitting Diodes," Journal of Electronic Packaging, vol. 132, no.3, 2010. doi: $10.1115 / 1.4002298$

[12] R. Hu, X.-B. Luo, H. Feng, and S. Liu, "Effect of Phosphor Settling on the Optical Performance of Phosphor-Converted White Light-Emitting Diode," Journal of Luminescence, vol. 132, no. 5. pp. 1252-
1256, 2012.

doi: 10.1016/i.jlumin.2011.12.059

[13] Philippe F. Smet, Anthony B. Parmentier, and Dirk Poelman, "Selecting Conversion Phosphors for White Light-Emitting Diodes," Journal of the Electrochemical Society, vol.158, no. 6, 2011. doi: 10.1149/1.3568524

[14] Z.-Y. Liu, S. Liu, K. Wang, and X.-B. Luo, "Optical Analysis of Phosphor's Location for High-Power Light-Emitting Diodes," IEEE Transactions on Device and Materials Reliability, vol 9, no. 1, pp. 65-73, 2009.

doi: $10.1109 / \mathrm{tdmr} .2008 .2010250$

[15] N. D. Quoc Anh, M.-F. Lai, H.-Y. Ma, and H.-Y. Lee, "Enhancing of Correlated Color Temperature Uniformity for Multi-Chip White-Light LEDs by Adding SiO2 in Phosphor layer," Journal of the Chinese Institute of Engineers, vol. 38, no. 3, pp. 299-303, 2014. doi: $10.1080 / 02533839.2014 .981214$

[16] M.-F. Lai, N. D. Quoc Anh, H.-Y. Ma, and H.-Y. Lee, "Scattering Effect of Sio2 Particles on Correlated Color Temperature Uniformity of Multi-Chip White Light LEDs," Journal of the Chinese Institute of Engineers, vol. 39, 2014. doi: $10.1080 / 02533839.2015 .1117950$

[17] Soni, Abhishek Kumar, and Vineet Kumar Rai, "Optical Studies in Er3 Doped BaMoO4 Downconverting Phosphor for Blue LEDs," 2016 doi: 10.1063/1.4945122

[18] J.-Y. Liao, Haibo Rao, Wei Wang, X.-L. Wan, L.-S. Zhou, Z. Da, X.-M. Wang, and Q.-L. Lei, “Optical Simulation of Phosphor Layer of White LEDs," Journal of Semiconductors, vol. 34, no. 5, 2013. doi: $10.1088 / 1674-4926 / 34 / 5 / 053008$

[19] Jana Ryckaert, Sven Leyre, Peter Hanselaer, and Youri Meuret, "Simulation of White LEDs with a Planar Luminescent Layer Using the Extended AddingDoubling Method," Optical Systems Design 2015 Illumination Optics IV, 2015. doi: $10.1117 / 12.2191165$

[20] J.-J. Zhong, M.-Y. Xie, Z. Ou, R. Zhang, M. Huang, and F.-L. Zhao, "Mie Theory Simulation of the Effect on Light Extraction by 2-D Nanostructure Fabrication," in proceeding of Symposium on Photonics and Optoelectronics (SOPO), Wuhan, China, May 16-18, 2011, vol. 1-4. doi: $10.1109 /$ SOPO.2011.5780566

[21] O. Tapaninen, "Optical and thermal simulation chain for LED package," in proceeding of 17th International Conference on Thermal, Mechanical and Multi-Physics Simulation and Experiments in Microelectronics and Microsystems (EuroSimE), 
Montpellier, France, April 18-20, 2016.

doi: 10.1109/EuroSimE.2016.7463349

[22] N. H. Khanh Nhan, T. H. Quang Minh, T. N. Nguyen, and M. Voznak, "Red-Emitting Ca2si5n8eu2+ Phosphor: A New Recommendation For Improving
Color Uniformity And Color Quality Scale Of The Conformal Packaging Multi-Chip White Leds" Journal of Ovonic Research, vol. 13, no. 6, pp. 325331, 2017. 